\title{
Forefronts in structure-performance models of separation membranes
}

\author{
Sourav Mondal ${ }^{\mathrm{a}, 1}$, Ian M. Griffiths ${ }^{\mathrm{a}}$, Guy Z. Ramon ${ }^{\mathrm{b}, *}$ \\ ${ }^{a}$ Mathematical Institute, University of Oxford, Oxford OX2 6GG, United Kingdom \\ ${ }^{b}$ Department of Civil \& Environmental Engineering, Technion - Israel Institute of Technology, Haifa, \\ Israel
}

\begin{abstract}
Polymeric membranes used for separations are complex porous structures, spanning lengths that range from truly molecular scales up to many micrometers. These porous structures can be characterized by both the spatial and size distribution of the pores, which can further be divided between the membrane surface distribution versus the depth distribution. Such structural features have a wide impact on membrane performance, ultimately dictating the fluid permeability and solute selectivity as well as the propensity for and severity of fouling. It is thus not surprising that in recent years significant emphasis has been placed on engineering the pore size and distribution to achieve a specific task. However, despite the technological progress, it is difficult to quantify the effects of membrane morphology on overall performance. Identifying advantageous porous structures would benefit design and motivate the development of better control over the formation of a given morphology. In this discussion, the relative importance of membrane morphologies, manifested as pore spatial and size distributions, is considered for several illustrative cases: pressure-driven flow as impacted by membrane surface pore locations and depth morphologies; the selectivity of a porous membrane as affected by a porosity gradient; and the performance of a composite membrane as impacted by the porous morphologies of support layer, as well as the film. Emphasis is placed on unifying concepts of mathematical modelling, as well as the link between theory and experimental observation. Finally, some future extensions needed for consolidating structure-performance
\end{abstract}

\footnotetext{
${ }^{*}$ Corresponding author

Email address: ramong@technion.ac.il (Guy Z. Ramon )

${ }^{1}$ Current address: Department of Chemical Engineering, Indian Institute of Technology Kharagpur, Kharagpur 721302, India
} 
models are mentioned.

Keywords: membrane transport; modelling; structure-performance; composite; porous structures; permeability; selectivity

\section{Introduction}

Membranes comprise complex porous structures; these function either as the actual separation medium, for example in micro/ultra-filtration, membrane chromatography and dialysis, or as a porous support structure that can impact the overall performance of the membrane, as in the case of composites used for gas separation, nanofiltration and reverse osmosis. Fig. 1 shows a collection of illustrative morphological features, highlighting the diversity in pore size and spatial distributions found in membranes (taken from refs [1-4]). These structural features can profoundly impact membrane performance, reflected by the fluid permeance and particle/solute selectivity. Performing exhaustive characterization of actual membrane performance as related to their structure can be time-consuming and costly, motivating the use of mathematical models with which to probe the large parameter space rapidly. Such models need not necessarily be fully predictive; rather, they should have the ability to pin-point the most important features and therefore guide actual fabrication efforts, leading to more promising avenues for improved membrane performance.

The aim of this discussion paper is to lay out the various ways in which porous structures influence transport through membranes in current industrial use, and to provide the theoretical framework behind the structure-performance analysis. In doing so, we attempt to concisely summarize existing theoretical work, extend some of the pre-existing theories, and highlight areas in which further work is needed to better understand and quantify membrane structure-performance. Where appropriate, we include published experimental verification of theoretical predictions. Furthermore, we highlight the need for interdisciplinary work, to combine the theoretical design strategies with real membrane application and development.

In order to create a generalized framework for the subsequent sections, we divide the structures to be considered into two broad categories, namely porous and composite membranes. A porous membrane possesses continuum-scale pores and transport through 
(a)

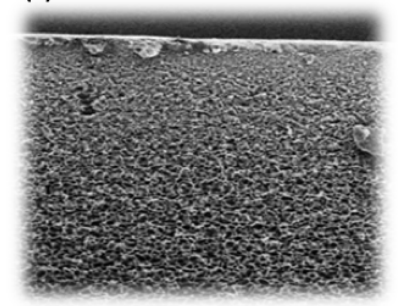

(e)

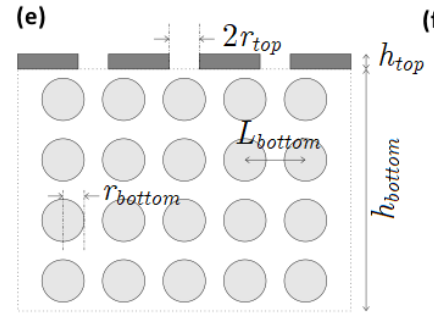

(b)

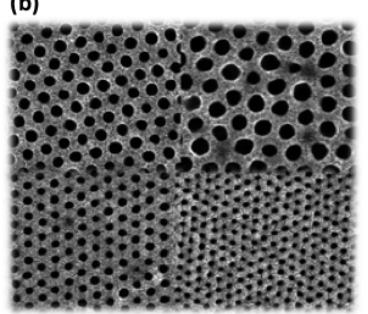

(f)

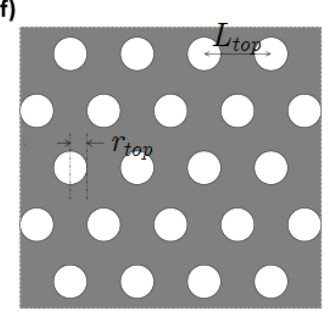

(c)

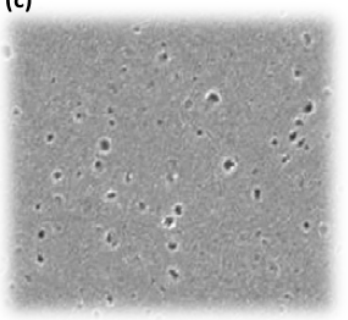

(g)

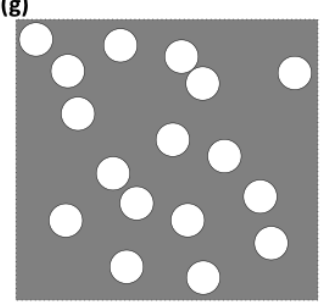

(d)

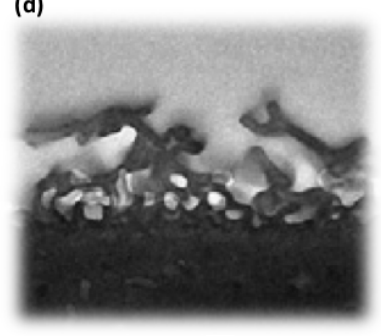

(h)

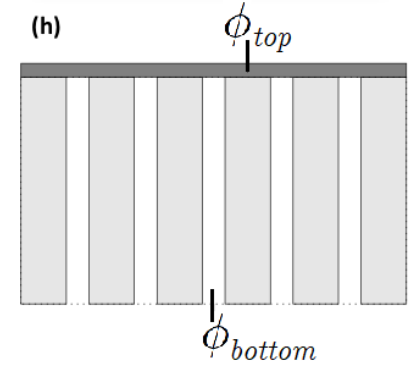

Figure 1: Membrane morphologies and model representation of a two-layer structure, as viewed from the top and cross-section. (a,e) cross-sectional view of typical phase-inversion UF membrane (image taken from [3] ) and the representation at a top, perforated sheet overlaying a bottom porous layer. $(\mathrm{b}, \mathrm{f})$ Top view of a porous membrane - periodic, block-copolymer phase inversion membranes, with tuneable pore size (image taken from [4]) and the representation as a perforated sheet with a hexagonal array (or square, not shown) of circular pores. (c,g) Top view of a porous membrane - typical phase-inversion membrane with random pore distribution, showing distinctive regions of high and low porosities (image taken from [1), and its representation as a perforated sheet with randomly located holes. (d,h) The interface between the polyamide 'active layer', or top film of a composite membrane, showing distinctive 'voids', and the underlying porous support (image taken from [2]). Shown below it is the idealized representation of a top film overlying a domain with alternating regions of the pores and solid matrix of the support.

it is governed by viscous flow (for the solvent), or advection and diffusion (for the solute). The mode of separation is by sieving at the interface or by capture within the depth. A typical example of a porous membrane is shown in Fig. 17. However, since in many cases the surface of the membrane possesses a structure that is different from the bulk material (for example, Fig. 1. vs. Fig. 1a), one may model the membrane as a simplified two-layer structure, shown schematically in Fig. 19: a top layer, describing the surface region, approximated as a perforated sheet (with different pore arrangements, as illustrated in Fig. 1f,g), and a bottom layer, describing the bulk material, composed of a porous medium. In this paper we shall consider cases in which both layers may affect membrane performance.

A composite membrane comprises a top-layer thin film and a porous support as the 
bottom layer, which may be composed of a different material (see image and schematic in Figs $1 \mathrm{~d}$ and $\mathrm{h}$, respectively). Unlike porous membranes, transport is considered to be predominantly diffusive and separation is almost entirely achieved by the top layer, normally an ultra-thin film of dense polymer [5, 6]. However, while the bottom layer is traditionally solely for structural support, its presence invariably plays a role in the overall performance and, as recently established, must be taken into account [7-10].

The structure of the paper is as follows. We begin by considering porous membranes in Section 2, We study the surface-versus-depth interplay and the effect of pore size and pore spatial distribution in the top layer. We compare cellular (or spongy) and granular structures and conclude by assessing the selectivity of porous membranes as impacted by porosity gradients. We then move on to composite membranes in Section 3 . We consider the effect of pore size and spatial distribution in the top and bottom layers, the relative effects of the top and bottom layer on performance, and conclude with a note on selectivity. Finally, in Section 4 we summarize the main points and conclusions, and also highlight future directions for further theoretical development, required to pave the way forward, improving the ability to determine desirable membrane structures, and linking them with fabrication methods.

\section{Porous membranes}

In this section we consider porous membranes, as previously defined; see Fig. 1e,f for a schematic and illustration of the nomenclature employed in this section. To fix notation, the permeance of a porous membrane is defined here as the flux achieved for a given pressure difference,

$$
k=\frac{Q}{A_{m} \Delta P}
$$

where $Q$ is the volumetric flow rate, $A_{m}$ is the membrane area and $\Delta P$ is the transmembrane pressure difference. This is related to the Darcy permeability via $k=\hat{k} / \mu h$, where $\mu$ is the fluid viscosity and $h$ is the membrane thickness (which may be attributed to the top layer, bottom layer or a combination). Note that $k$ is identical to the commonly used notation of $L_{p}$ (or, as often used for NF/RO membranes, $A$ ).

In what follows, we examine how permeance is affected by: (i) surface and depth porosity; 
(ii) pore-size distribution in the top layer; and (iii) pore morphology. We conclude by examining the selectivity-permeance trade-off. Specifically, we begin by studying the viscous flow through a porous membrane, as affected by pore morphology, considering the separate effects of the entrance and the 'depth'. This is followed by the analysis of the permeance of contrasting morphologies resulting from the phase-separation process used for membrane fabrication. Finally, we consider the selectivity of a membrane in the presence of a porosity gradient.

\subsection{Surface versus depth effect}

The flow through a porous layer, in its simplest form, may be envisioned as that through a bundle of capillaries, where in each individual 'tube' one has a fully developed, viscous Poiseuille flow. It is also a common, simplified, model for porous membranes, with pores of radius $r$ and length $h$ (representing the membrane thickness as well), and corrections introduced for the effective pore area and length, via a tortuosity and surface porosity [5, 6]. However, while normally neglected in this common modeling framework, the topmost section of the porous layer, where the fluid transitions from the free domain to the confinement of the pores, may represent a significant mechanism of energy dissipation, contributing to the overall pressure drop.

Neglecting surface effects is likely to be a good approximation when, indeed, the resistance to flow is dominated by the bulk. However, as membranes strive to become thin or ultra-permeable, surface effects are expected to become important. Examples include increasingly thinner 'skin' layers in phase-separated membranes (e.g., [4, 11, 12]), ultra-thin silicon membranes [13, 14], carbon-based membranes, e.g., nanotubes [15] or graphene oxide [16] as well as biomimetic water channels [17]. In particular, carbon-nanotube and water-channel-based membranes can pose an interesting scenario, where a thick bulk material poses a very small resistance to the flow [18]; this, again, would lead to a situation where most of the pressure drop through the membrane structure occurs at the surface, near the pore entrance. This has recently been modelled on the scale of a single nanotube, using molecular dynamics simulations, illustrating that over $90 \%$ of the flow resistance is at the nanotube entrance [16, 19]. A similar effect has also been shown in a recent computational study, where artificial structures were modelled in 3D, comparing the flow through a purely porous membrane to that of a membrane with a bi-layer structure - a 
porous top and finger-like voids dominating the bottom [20].

In light of this question, the current section considers transport through a conceptual bilayer structure consisting of a thin perforated sheet, or 'skin', overlaying a porous layer (shown schematically in Figs. 22-f). We evaluate the relative importance of this 'surface' contribution to the overall resistance to flow. In order to account for the effect of the top layer, one may resort to the simplest representation, proposed by Weissberg [21], in which the flow through the membrane is assumed to be the superposition of a flow through a surface pore of radius $r_{\text {top }}$ in an infinitely thin plate (Sampson flow [22]) and the flow through an individual cylindrical tube of length (thickness) $h_{\text {bottom }}$ (Poiseuille flow, 223]). Expressed as the permeance (using Eq. 1), this gives

$$
k=\frac{1}{A_{m}} \frac{\pi r_{\text {top }}^{3}}{\mu\left[3 \pi+\left(8 h_{\text {bottom }} / r_{\text {top }}\right)\right]},
$$

for an isolated pore. In Eq. (2) we observe the two contributions to the pressure drop: the first term in the denominator corresponds to surface effects, while the second term corresponds to the depth. When the thickness to pore-size is large $\left(h_{\text {bottom }} / r_{\text {top }} \gg 1\right)$ the flow is predominantly Poiseuille, while for a thin, dense skin region (top layer) when $h_{\text {bottom }} / r_{\text {top }} \ll 1$, Sampson flow dominates.

Eq. (2) represents the simplest combination of surface and depth effects. However, a more comprehensive model requires a full solution of the flow problem, where both contributions are accounted for concurrently. To this end, we solve the steady-state Stokes equations, within a model geometry, as shown schematically in Fig. 2a, depicting a bottom layer composed of spherical obstacles, connected to a top, 'free' fluid domain through a thin perforated sheet (see Appendix A for details). We note that this is by no means a comprehensive representation of a membrane structure, but it offers a relatively convenient, self-consistent way of assessing the impact of morphology.

The plots shown in Fig. 2 illustrate that the top-layer resistance may play a significant role in the overall permeance of the membrane, in particular when the porosity of the bottom layer, $\phi_{\text {bottom }}$, is high. For example, when $\phi_{\text {bottom }}$ is fixed at $80 \%$, the permeance when the porosity of the top layer, $\phi_{\text {top }}=30 \%$ is only $43 \%$ of that when $\phi_{\text {top }}=100 \%$ (see Fig. 2b). As we might anticipate, the permeance is limited by the top layer porosity $\left(\phi_{\text {top }}\right)$ when $\phi_{\text {bottom }}$ is high (Fig. 2b). These results suggest that membrane design must consider both these effects for proper optimization; this is expected to be particularly so for some 
(a)

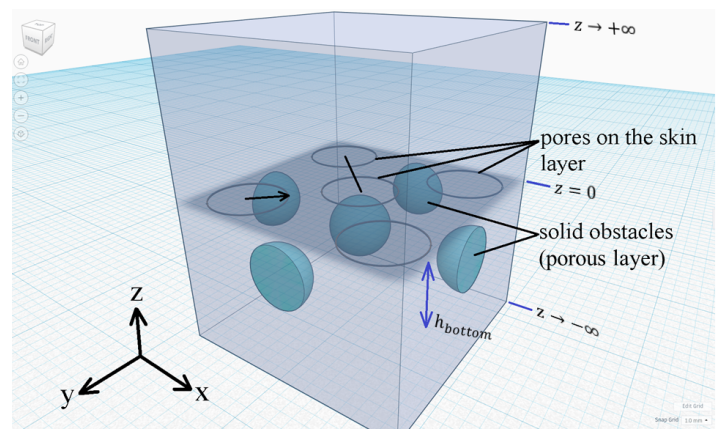

(b)

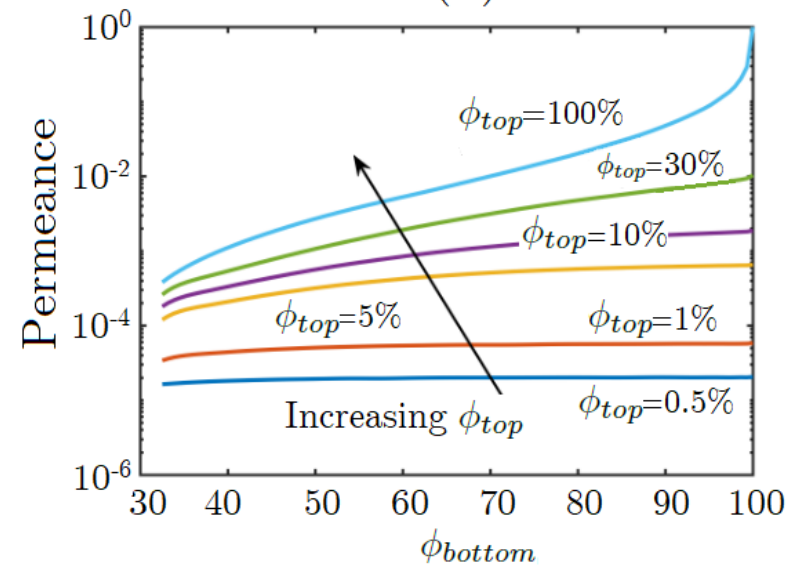

Figure 2: The effect of surface and bulk porosity on the membrane permeance. (a) Schematic of the model configuration. An array of spheres is used to model the 'depth' porous structure, with porosity $\phi_{\text {bottom }}$. This layer is connected to a fluid reservoir through a perforated sheet with a surface porosity $\phi_{t o p}$. The equations for Stokes flow are solved within this configuration, from which the permeance is calculated (see Appendix A for details of the calculation). (b) The permeance as a function of the bottom layer porosity, $\phi_{\text {bottom }}$, calculated for varying surface porosities. Here, we take $\tilde{h}_{\text {top }}=0$ and $\tilde{h}_{\text {bottom }}=1$.

high-performance structures mentioned previously, such as ultra-thin membranes, CNTbased structures, and biomimetic water-channels - the resistance through all of which is expected to be surface-dominated.

\subsection{The effect of pore size and location distributions in the top layer}

In the previous section it was illustrated that the resistance to flow imposed by the surface pores can become important for realistic structures and, particularly, for thin selective layers or low-resistance bulk materials. However, the calculations were made for a squarearray periodic lattice while, for most membranes in current industrial use, the surface pores are not evenly distributed, and are not of the same size (or, indeed, shape) - see Fig. 1c for a representative image. Of these, the effect of size distribution has received the most attention, particularly due to its influence on selectivity through mechanical sieving (see, for example, [24, 25]). Shape effects have also been considered, particularly slit-shaped pores versus circular pores [26]. However, these permeance calculations still considered the membrane as a bundle of cylindrical capillaries, ignoring the surface effect. Here, we consider the effect of pore size distributions on the resistance of the top layer; we then calculate the permeance as affected by the spatial distribution of pores, for two periodic arrays and, more importantly, a random distribution of pore locations. First, we 
consider the simple generalization of the surface contribution in Eq. (2) that accounts for a given pore size distribution, $f\left(r_{t o p}\right)$, which was obtained by Jensen et al. [27],

$$
k=\frac{M_{3}}{3 \mu A_{m}},
$$

where $M_{3}$ is the third statistical moment of the pore size distribution. The log-normal density function has been used extensively to describe membrane pore-size distributions (see, for example, [28]), and it assumes that the natural logarithm of the pore

radius is normally distributed, $f\left(r_{\text {top }}\right)=\frac{1}{r_{\text {top }} \sigma \sqrt{2 \pi}} \exp \left[-\frac{\left(\ln \left(r_{\text {top }}-r_{\text {top }, a v g}\right)\right)^{2}}{2 \sigma^{2}}\right]$ so that $M_{3}=$ $r_{\text {top,avg }}^{3} \exp \left(3 \sigma^{2}\right)$, where $r_{\text {top,avg }}$ is the mean pore size. In Fig. 3 a we consider the effect of the pore size distribution (for a square array) characterized by the standard deviation, $\sigma$, on the flow through the top thin sheet, showing the impact of porosity on the permeance. We observe that the membrane permeance increases with the pore size variability. This is due to the fact that the presence of larger pores increases the flux quadratically with the pore radius and so strongly impacts the permeance. However, this must be traded against the fact that higher variability in pore sizes also reduces the selectivity of the membrane, since larger solutes or particles are now able to pass.

Next, we consider circular pores of a fixed radius $r_{\text {top }}$ and vary their spatial distribution. For periodic square or hexagonal arrays, a generalized expression of Eq. (2) for the pressure drop is given by [27],

$$
k=\frac{r_{t o p}^{3}}{3 A_{m} \mu\left[1-\left(r_{t o p} / L_{t o p}\right)^{3} \xi\right]},
$$

where $L_{t o p}$ is the inter-pore distance to the nearest neighbor (as seen in Fig. 1f) and $\xi$ is a constant depending only on the geometrical layout of the pores in the layer; an exact expression exists for $\xi$ in the form of an infinite series, giving $\xi \approx 1.9$ for a square lattice and $\xi \approx 2.3$ for a hexagonal lattice [27]. The term containing $\xi$ accounts for the flow through pores when they are closer to each other and no longer isolated, in which case, they hydrodynamically interact, i.e., the flow through one pore affects the flow through the neighboring pores. This interaction yields a larger flow per pore than that predicted by the classical Sampson result for an isolated pore having an identical pressure drop (compare Eq. (4) with Eq. (2)). In Fig. 3b we compare the analytical prediction (4) with numerical simulations for a range of top-layer porosities, $\phi_{\text {top }}$ in either a square or hexagonal array (see Appendix B for details). Good qualitative 
(a)

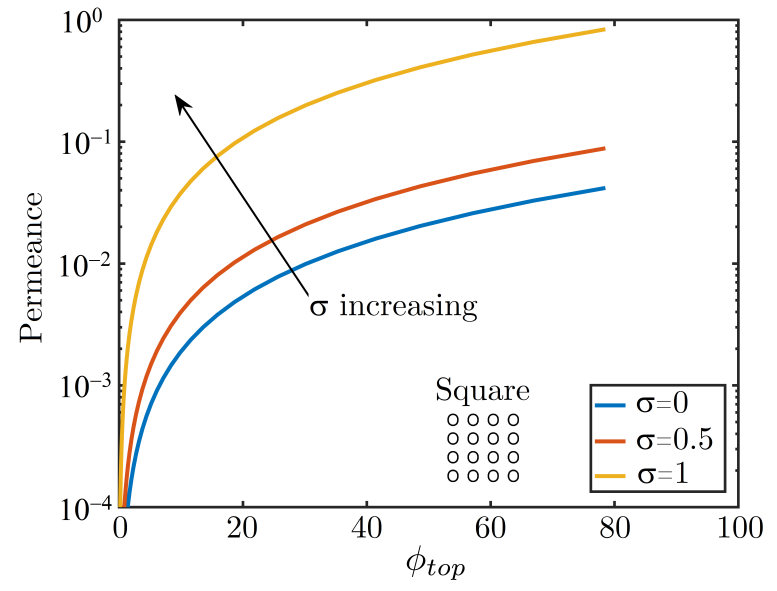

(b)

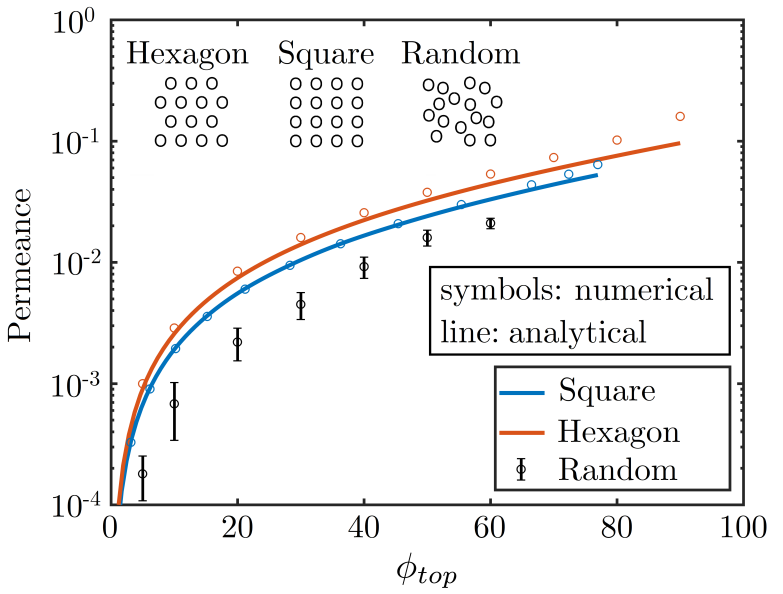

Figure 3: The effect of surface pore size and spatial distributions on the permeance of an infinitely thin, perforated sheet, representing the 'surface effect' of a membrane. (a) The permeance as a function of $\phi_{\text {top }}$, calculated for a square array of pores with size distributions of different variance, $\sigma$. (b) Calculated permeance vs. the top layer porosity, $\phi_{t o p}$, for a uniform pore size, spatially distributed as either periodic square and hexagonal arrays, or as a random distribution. Numerical calculations are detailed in Appendix B; the analytic calculations are based on [27. For the random distribution, there are 30 simulations (samplings) for each point and one standard deviation is represented by the error bars (see Appendix B for further explanation). Note that the curves representing $\sigma=0$ in (a) and the square pore array in (b) are the same and so give an idea of the connection between the two figures (both lines are blue in the color version).

agreement is observed, with deviation only for high porosities (when $r_{t o p} / L_{t o p}$ is not so small). However, in practice it is more difficult to manufacture regular arrays so an interesting question is - how much do we lose in permeance, when the pore arrangement is not regular? To this end we also compute, numerically, the permeance of a random arrangement of non-overlapping circular pores and include this in Fig. 3p (see Appendix B for details).

We find that the hexagonal lattice yields the highest permeance, with a small drop for a square lattice and a more substantial drop for a random distribution. The reason for this is that, in the random case, pores can be bunched together, creating areas of impermeable space on the surface. This implies that the fluid approaching the membrane in these areas must be significantly diverted to reach the pores, which leads to additional dissipation and, hence, reduced permeance. Periodic distributions out-perform the random case by at least a factor of two, for all porosities $\phi_{t o p}$. The relative increase in permeability offered by periodic distributions becomes even greater at higher porosity (note that Fig. 3b is on 
a logarithmic scale). Consequently, this indicates that it is well worth investing resources in manufacturing membranes with regular pores and high porosity.

\section{3. 'Cellular' versus 'granular' structures}

A commonly used process for fabrication of porous membranes is phase-separation, which may be induced by several techniques. Such membranes may generally evolve into two distinctive structures: (1) a cellular structure, in which the nucleating droplets during the phase-separation process are the polymer-poor phase, or (2) a granular structure where the solid phase of the membrane comprises the polymer-rich droplets and the membrane resembles a porous bed of spheres [5]. Fig. 4 a presents two representative images showing PVDF membranes fabricated using two non-solvents, leading to either a cellular or granular structure [29]. It seems that the cellular structure is more commonly found in commercial membranes, although recently developed block-copolymer membranes appear to possess an inherently granular structure [12, 30].

In light of the possibility to manufacture both structures, a question, yet to be considered, is which would be better in terms of the resulting permeance. We therefore turn to model the viscous flow through structures that mimic a cellular/granular morphology (schematically shown in Fig. $4 \mathrm{~b}$ ). We note that, when simulating the cellular structure, the diameter of the sphere representing the void is larger than the edge length so that there is an overlap with neighboring periodic spheres to ensure inter-connectivity and continuity of the resultant flow path, which places a limit on the range of physically valid porosities. Here, only the flow through the bottom layer is considered, and the entrance effect is not accounted for. Further details of the performed calculation may be found in Appendix C.

An illustrative calculation is shown in Fig. 4c for the cellular and granular morphologies. The results show that a granular structure has a better permeance than a cellular structure for a given porosity. This could have interesting implications if a granular structure could, from a solid-mechanics perspective, potentially offer a more robust structure in terms of compaction. Such properties may help reduce the deformation undergone by membranes upon exposure to the applied pressure, particularly support membranes for composites used in high-pressure applications. We note that, since the calculation is made for a repeating, periodic cell, it cannot fully account for the 
complexity of a real-life structure. However, it should provide a qualitative indication of the general effect that these morphologies are expected to have on the resistance to viscous flow. More refined calculations and comparison with careful experiments are needed in order to shed further light on these questions.

\subsection{Selectivity of porous structures}

The main mode of selectivity for porous membranes is size-based mechanical sieving. While other mechanisms, for instance, electrostatic interaction or Brownian motion, may become important under certain conditions, these involve material-specific physicochemical properties and are beyond the scope of the current discussion (an example of poreentrance effects may be found in [31], while hindered transport within the pore structure has been considered extensively, with a good overview given in [32, 33]). The selectivity is normally represented as a function of the size ratio between the particle being rejected $\left(r_{p}\right)$ and the membrane pore radius $(r), \lambda \equiv r_{p} / r_{\text {top }}$. Early work by Ferry [34] considered the pure effect of volume exclusion on the rejection of a rigid sphere by a cylindrical pore, yielding the following, simple yet remarkably representative, relation for the ratio of the concentrations outside and inside the pore, or sieving coefficient,

$$
S=(1-\lambda)^{2}\left[2-(1-\lambda)^{2}\right]
$$

(a)

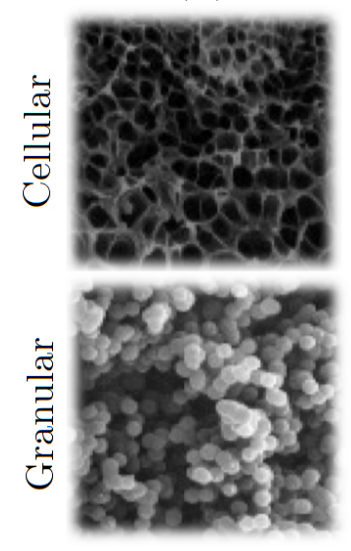

(b)

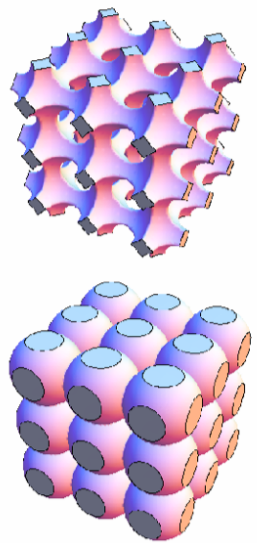

(c)

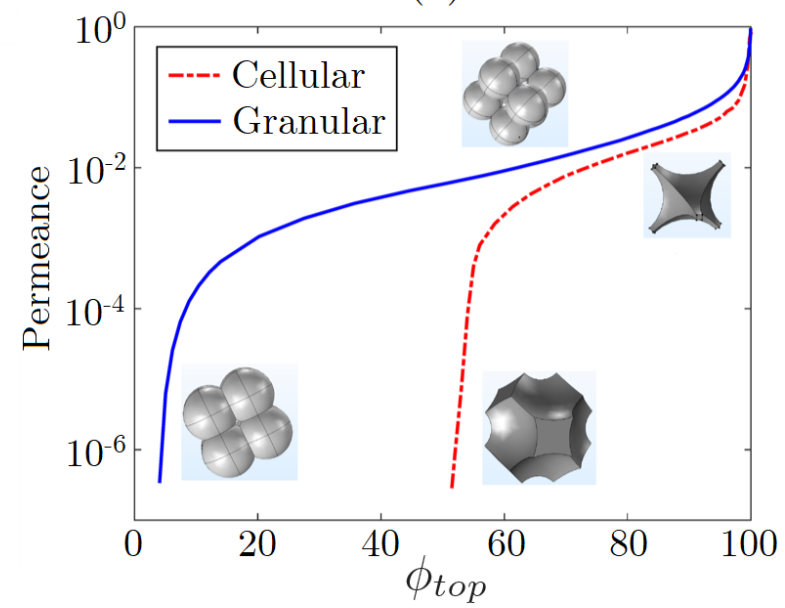

Figure 4: (a) 'Cellular' vs. 'Granular' morphologies of a PVDF membrane fabricated using the common nonsolvent-induced phase separation technique [29]. (b) Different morphological configurations of the 'bottom' layer of a porous membrane, with cubic lattice arrangement of the voids or obstacles (to create the granular or cellular matrix respectively). In this figure, $\phi_{t o p}=100 \%$ so the top layer does not exist.

(c) Calculated permeance vs. porosity for the two morphologies. 
As already mentioned in the previous section, most commercial membranes do not have a uniform surface pore size and exhibit a distribution of pore sizes. To determine the significance of the pore size distribution on the sieving of a given particle size, one may integrate over all possible ratios $\lambda$ to find an effective sieving coefficient. Variants of Eq. (5) have been developed over the years but the differences may be considered as refinements, such as a strategy for estimating $\lambda[24]$.

A natural outcome of this retention mechanism is a trade-off between the permeance of a membrane and its separation capacity [25]. Fig. 5a illustrates this, comparing a collection of commercial membranes and calculations based on a simple sieving model, for a log-normal pore size distribution, as well as isoporous membranes. As may be seen, the selectivity-permeance trade-off has a different characteristic shape, at a given mean pore size, dependent on the distribution variance, $\sigma$ (see different colors in Fig. 5 a). This curve is shifted to the right, increasing the permeability at a given selectivity, with a decrease in the parameter $h_{t o p} / \phi_{t o p}$, the ratio of the skin layer thickness and its porosity. For a given thickness and porosity, isoporous membranes retain higher permeance as the mean-pore size decreases (and selectivity increases). However, the experimental data shows that most isoporous membranes reported so far, do not outperform 'conventional' membranes; as is clearly seen, the key to improving the trade-off curve, particularly for isoporous membranes, lies in the reduction of the skin thickness and increasing the surface porosity (see, also, [12, 30]).

In the framework described above, the sieving is considered to occur solely at the pore entrance, so particles that enter the porous layer are assumed to be carried through the entire membrane and into the permeate. However, in practice, a particle may be slowed down and possibly captured within the depth of the membrane. While the case of hindered transport through a pore is a classical problem in the membrane literature (see the review by Deen [32] and references therein), it is not commonly used for assessing membrane selectivity, presumably due to the complexity of the calculation and required intimate knowledge of the pore structure. More importantly, the case of solute/particle uptake in the presence of a porosity gradient has received far less attention. Conceivably, the additional possibility of capture within the membrane may provide a route to modifying the usual permeability-selectivity trade-off curve, albeit with an inherent risk of fouling (which also exists with external sieving, but here will become internal). This 
becomes particularly interesting when considering cases where the membrane possesses a depth gradient in porosity. Specific applications such as membrane chromatography, where such capture is at the core of the process, can likely benefit from this design consideration. For this case, which is essentially depth filtration, the effect of a porosity variation across the depth of the filter medium (the membrane) can be studied in detail using the method of homogenization, which can account for microstructural details and yet produce a model that considers the transport through an 'effective' medium, therefore significantly reducing the computational cost [35] (see Appendix D). Indeed, as is seen in Fig. 5b, where the selectivity is plotted against the permeance for a membrane with a unifom pore size but with varying porosity and porosity gradient, one has a family of possible curves that modify a given single curve one obtains when only surface sieving is accounted for. Furthermore, the calculations illustrate that a positive gradient in porosity (porosity increasing with depth) provides better selectivity at a given surface porosity. This additional depth contribution allows a further tweak of the membrane selectivity at a given surface pore size; needless to say, such control over the depth structure is non-trivial, and these results merely serve as the motivation to seek the means by which such structures might be achieved in practice. 
(a)

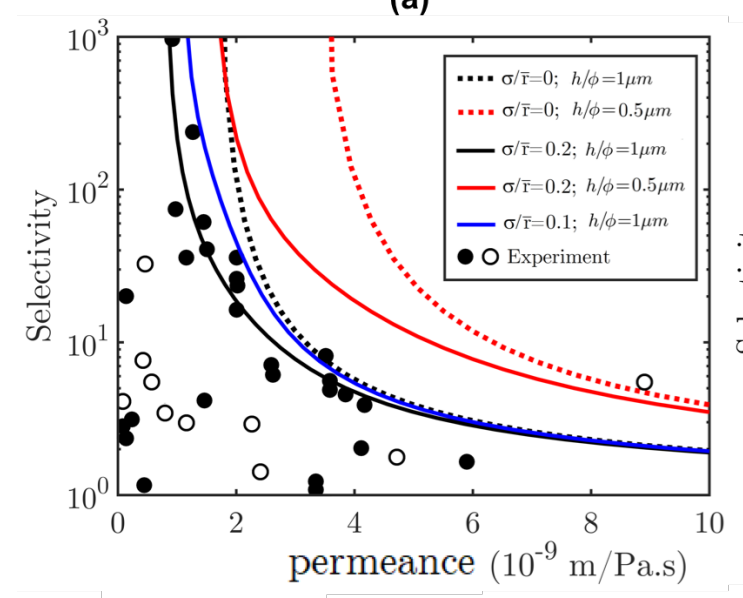

(b)

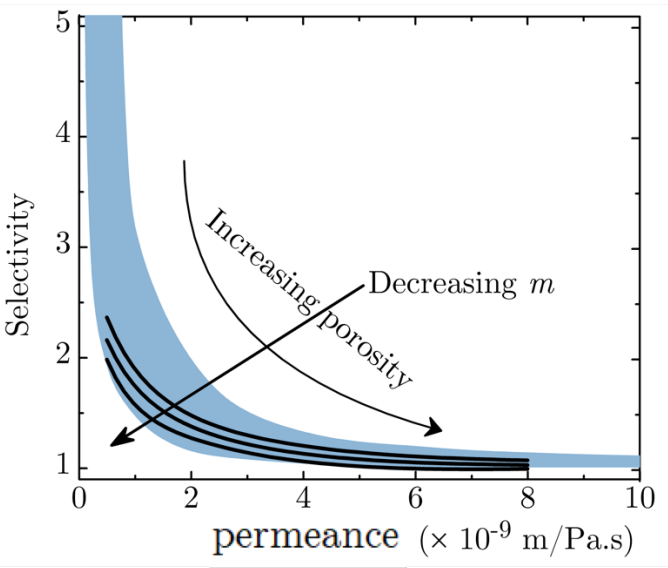

Figure 5: (a) 'Surface' selectivity vs. permeance. Experimental points from the literature, with empty symbols representing isoporous membranes (taken from [12]). Lines are calculations based on a simplified model that accounts for mechanical sieving at a pore entrance and Poiseuille flow through straight cylindrical pores (see, e.g. [25]). The even-colored lines represent membranes with equal skin layer thickness/porosity ratios ( $h / \phi$, each representing the 'top', in this case), while dotted vs. solid curves represent isoporous $(\sigma=0)$ and log-normal distributed pore size distributions, respectively. (b) 'Depth' selectivity vs. permeance, the consequence of capture within the membrane structure, calculated for a varying depth porosity $\phi_{\text {bottom }}(z)=\phi_{0}+m(z-0.5)$. The parameters $m$ and $\phi_{0}$ represents the porosity gradient and initial porosity respectively, which are varied proportionately so that the value of $\phi_{\text {bottom }}$ is within the theoretical bounds of $[1-\pi / 6,1]$ in three dimensions (see details in Appendix D). 


\section{Composite membranes}

Membranes used for reverse osmosis, as well as for gas separation, are composite structures comprising a porous support over which an ultra-thin film is fabricated, which serves as the primary selective layer (often referred to as the 'active' or 'barrier' layer) [5, 6]. The support, generally assumed to provide structural integrity alone, is usually a porous ultrafiltration membrane. Transport through the thin film occurs by a solution-diffusion mechanism [36, 37], which is assumed to govern all permeating species separately, i.e., for the case of desalination, water and salt transport is uncoupled. While recognized quite early and investigated by several researchers [38 41], the effect of the support on the overall transport of a composite appears to have begun receiving much-needed attention only recently [7-10, 42,47]. Furthermore, in the case of polyamide thin films used for desalination, recent evidence shows that the top layer is not homogeneous and contains voids [48 51], which have been shown to be liquid-filled under normal operating conditions [48. These features, mostly of a geometrical nature, are not accounted for in the classical model, where it is implicitly assumed that the membrane is a flat, homogeneous layer, and that the solute and solvent are assumed to diffuse independently, with their transport governed by the diffusion equation.

In the following section we examine how the overall permeability of the composite structure is affected by the support layer properties, namely pore morphology and solute diffusivity within the solid-phase, as well as the porous nature of the thin film. We conclude by commenting on the effect of structure on the selectivity-permeance trade-off.

\subsection{Effect of the support on transport through composites}

The porous support, generally made from an impermeable solid matrix, creates an obstruction to the transport out of the top, dense, active layer; it has so far been generally assumed that only the pores at the support-film interface allow transport and so, theoretically, the overall permeance of the composite membrane is always lower than that of the unsupported thin film. This effect has recently been confirmed experimentally for composite membranes used in both gas separation and desalination [9, 10]. In the case of desalination membranes, thin films, created through support-free interfacial polymerization, were placed on supports possessing different permeances, illustrating a clear 
correlation between support permeance and the overall composite permeance, as shown in Fig. 6a [10]. A similar approach was used to demonstrate the effect of support porosity and film thickness in the case of gas-separation membranes, shown in Fig. 6b [9]; here, the experimental results also compared well with theoretical predictions.

To illustrate the main effects leading to the support impact on overall transport, we first state the general mathematical problem underlying the modelling attempts made so far in the literature [7, 8, 38, 40, 44]. Transport through the top layer, according to the solution-diffusion model, consists of an initial stage where the solvent or solute partition into the polymer phase, followed by diffusion along a gradient in the chemical potential. Furthermore, the flux of the various components of the mixture is assumed to be decoupled [36, 37]. While the gradient in chemical potential may be due to pressure or concentration, dependent on the application, we follow the compositional distribution of a diffusing species along the depth of the top layer, governed by the Laplace equation,

$$
\nabla^{2} C=0
$$

in which the concentration, $C$, is scaled with respect to the feed and permeate streams (subscripts $f$ and $p$, respectively), via $C=\left(c-c_{p}\right) /\left(c_{f}-c_{p}\right)$. The boundary condition applied on the feed side is, simply, $C=1$. However, the bottom of the film may be in contact either with the fluid within a support pore, in which case the boundary condition is a perfect sink,

$$
C=0
$$

or with the solid phase of the support material, where a no-flux condition has commonly been imposed, i.e.,

$$
\mathbf{n} \cdot \nabla C=0
$$

on the solid support material, considered to be impermeable to the diffusing species, with normal $\mathbf{n}$. The assumption of non-penetration into the support solid material may be relaxed, as will be discussed below. We also note, in this context, that while it has been shown that the support impacts transport, there is no direct experimental evidence on the degree of transport hindrance manifested by different support materials, in the solid phase. Finally, a periodic boundary condition is imposed on the remaining boundaries, forcing symmetry. This applies to periodic pore 
arrangements; for details of the calculations in the case of random pore distributions, please refer to Appendix E.

The effective properties of the composite membrane, such as its permeance, will depend on the thin-film thickness $\left(h_{t o p}\right)$ and the pore size of the support $\left(r_{\text {bottom }}\right)$, as well as its porosity $\left(\phi_{\text {bottom }}\right)$. A previous study has shown that permeance is maximized when increasing the number of pores at a constant porosity, i.e., having many small pores is better than having fewer large pores [7].

An empirical relation of the relative permeance $\left(k_{r}\right)$ - the permeance of the composite relative to that of the thin film in the absence of the support layer - has been obtained by Wijmans and Hao [8] from a fit to data from numerical calculations,

$$
k_{r}=\frac{\phi_{\text {bottom }}+1.6\left[\frac{h_{\text {top }}}{r_{\text {bottom }}}\left(\frac{\phi_{\text {bottom }}}{1-\phi_{\text {bottom }}}\right)\right]^{1.1}}{1+1.6\left[\frac{h_{\text {top }}}{r_{\text {bottom }}}\left(\frac{\phi_{\text {bottom }}}{1-\phi_{\text {bottom }}}\right)\right]^{1.1}} .
$$

This correlation predicts the restriction factor within $5 \%$ of the numerical calculations for all cases evaluated, which included three different pore distribution patterns and multiple pore size variations with up to four different pore sizes. An analytic solution to this problem has also been derived, using asymptotic methods, assuming $r_{\text {bottom }} / h_{\text {top }} \ll 1$ [44]

$$
k_{r} \approx \frac{4 r_{\text {bottom }} h_{\text {top }}}{L^{2}+4 h_{\text {top }} r_{\text {bottom }}-4 r_{\text {bottom }} \epsilon G(0)},
$$

where $L$ is the inter-pore spacing and $G(0) \simeq 0.62$ is the Green's function at the origin (see appendix in [44). Eq. 10 matches well with the numerical predictions when $r_{\text {bottom }} / h_{\text {top }}<$ 0.2. It is naturally useful to have analytic expressions from which it is straightforward to calculate projected properties, rather than perform the full numerical calculations.

We now turn to consider another important aspect: the distribution of pore size and spatial location. Previous modelling studies have assumed periodic pore arrangements, which clearly do not represent the random pore distributions observed in commercially used supports (see, for example, Fig. 1. for foresentative image of a polysulfone support membrane). Within a periodic array, the effect of pore size variation has been examined, to some extent, by Wijmans \& Hao [8], showing that having pores of different sizes on a periodic lattice made a very small impact on the permeance. Here, we further extended the model to incorporate the spatial distribution of the pores in the support, calculating 
(a)

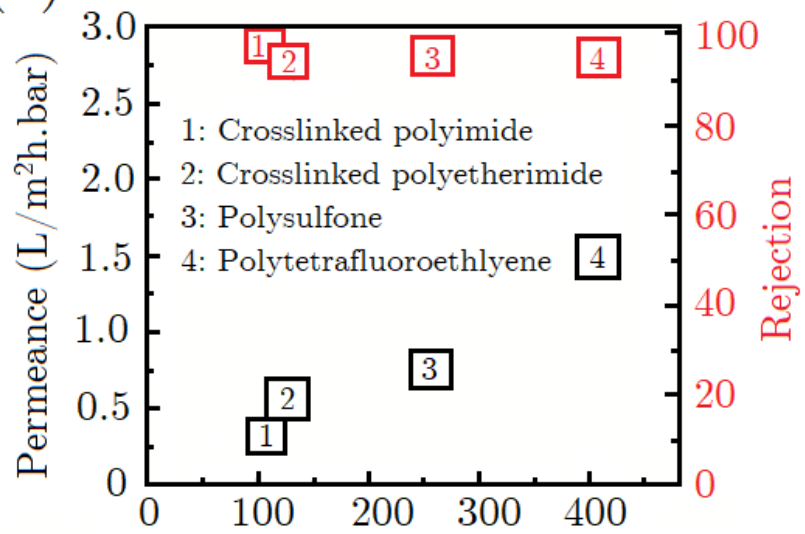

Support permeance $\left(\mathrm{L} / \mathrm{m}^{2} \mathrm{~h}\right.$. bar $)$

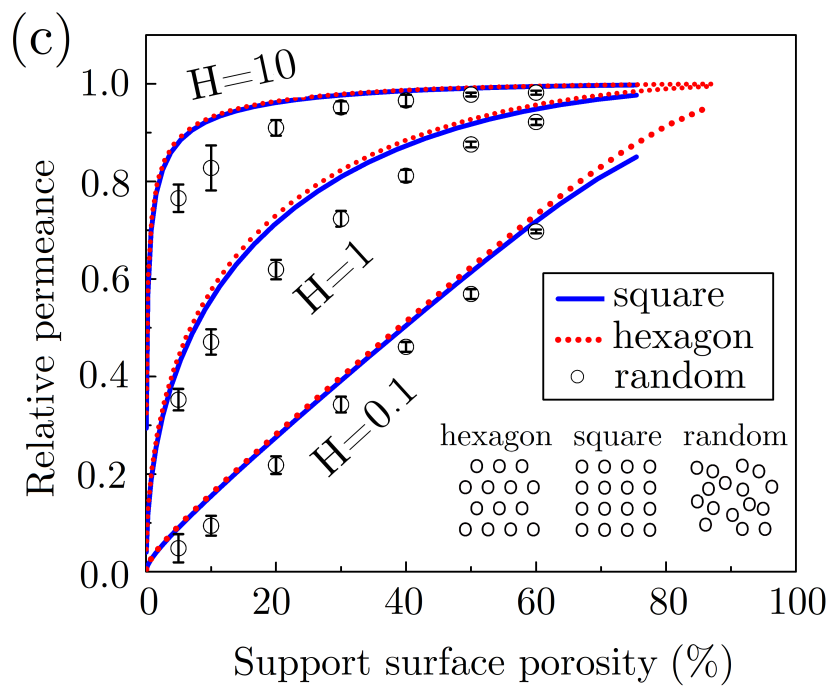

(b)

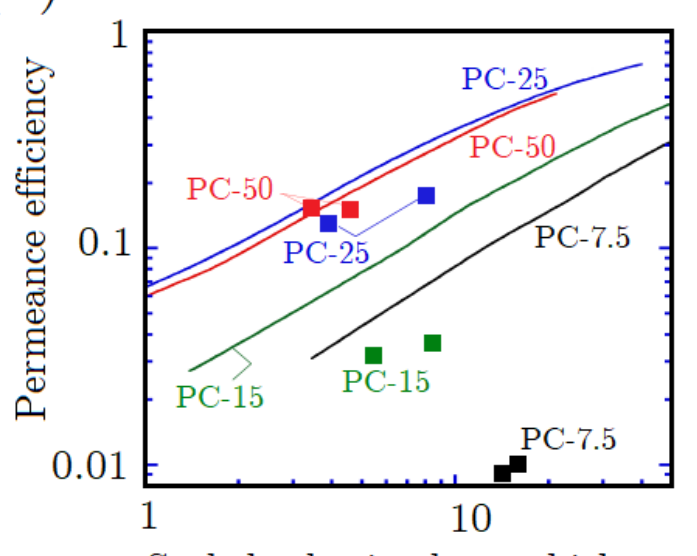

Scaled selective layer thickness

(d)

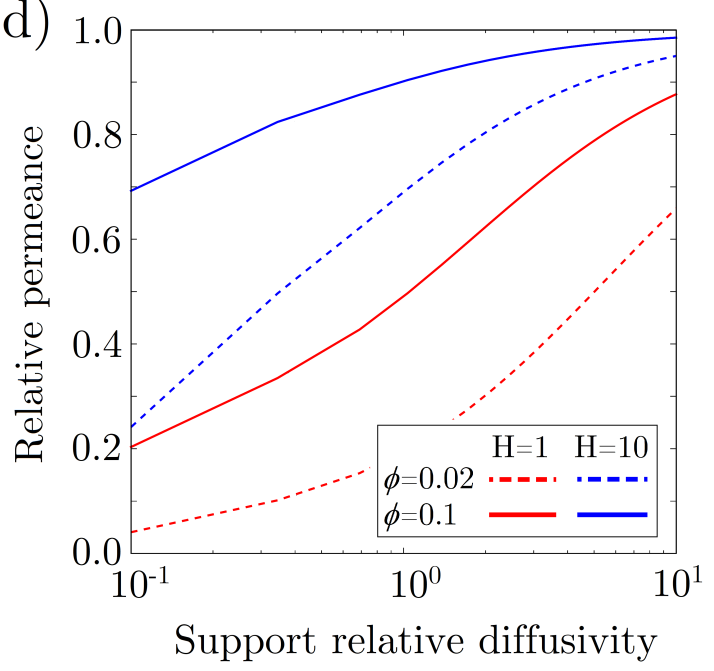

Figure 6: The effect of support pore morphology on transport through a composite membrane. (a) Experimentally measured permeance of thin-film composite desalination membranes, where an identical polyamide membrane was placed on supports with varying permeance. Taken from [10]. (b) The relative permeance (scaled against that of the free-standing thin film) of a gas-permeation composite, shown for supports with different porosities, as a function of the film thickness, scaled against the pore size $\left(H=h_{\text {top }} / r_{\text {bottom }}\right)$. Solid curves are theoretical predictions. Taken from [9]. (c) Model calculations showing the variation of the relative permeance with support porosity, for different scaled top-layer thicknesses. Calculations made for two periodic arrangements of the support pores, as well as a random surface distribution. The error bars represent one standard deviation and 50 simulations (samplings) are done for each point. (d) The effect of transport through the solid phase of the support material on the relative permeance, shown in terms of the ratio of the diffusivity in the the support material vs. the diffusivity in top film. Calculations shown for two support surface porosities and scaled film thicknesses. Taken from [7.

the relative permeance for random pore locations, compared with that obtained for two representative periodic distributions (see Fig. 6c). 
We note that as the porosity increases, the standard deviation of the mean value (the data points in Fig. 6c) decreases, attributed to the lower available area for the sampling (see Appendix B).

The main outcome of the foregoing analysis is that a composite structure will always have a lower permeance than the free-standing thin film, due to obstruction by the support when the support material has a permeability much smaller than that of the 'active layer' (which has been assumed to be the case for supports used in NF/RO and gas separation [7, 8, 10, 45]). This obstruction and consequent reduction in permeance becomes more severe as the ratio of film thickness to pore size decreases, at a given porosity (or, conversely, as pore size increases while the film thickness is fixed). Another interesting feature is that the periodic pore structures in the top layer of the support membrane show very little difference between square and hexagonal arrays (the latter being slightly better) and produce a higher permeance than the random distribution. However, the increased permeability when shifting from a random to a periodic pore arrangement is not very large and is, for the most part, smaller than $10 \%$ and, at most, $20 \%$ (see Fig. 6c). This may shed some doubt on the motivation for using periodic supports, should they prove more expensive and complicated to produce. The advantage of such membranes may still be found in the event that the overall surface porosity would far exceed their random counterparts, with small pore size: this is the best strategy suggested so far for maximizing the overall permeance [7, 8].

On the other hand, a strategy that has theoretically shown a large potential impact on the permeability is the fabrication of the composite using a support featuring a solid that is more permeable to the solvent (water, say, in the case of desalination membranes) than is currently used [7]. In this case, the solid fraction (the grey shaded pillars in Fig. 1 $\mathrm{h}$ ) is partially diffusing instead of a non-penetration boundary, and can be transformed by modifying the flux continuity boundary condition as [7]

$$
D_{f} \nabla C_{f}=D_{s} \nabla C_{s}
$$

where $D$ is the diffusivity of the species, with subscripts $f$ for the film and $s$ for the support region. This condition distinguishes the impact of the permeable support layer on the overall permeability of the composite membrane. The influence of the relative support diffusivities $\left(D_{s} / D_{f}\right)$ on the permeability is depicted in Fig. 6d. In the limiting 
case of $D_{s} / D_{f} \rightarrow 0$, the results correspond to the case of an impermeable support as shown in Fig. 6. The figure strongly indicates that the support diffusivity significantly enhances the overall permeability compared with the case of an impermeable support. However, the effect of support diffusivity is gradual (with increasing diffusivity) for smaller film thickness, comparing the curves for $H=1$ and $H=10$, where $H=h_{\text {top }} / r_{\text {bottom }}$ is the ratio of the film thickness to support pore radius. Substantial improvement of the overall permeance is already observed when the transport through the solid support is comparable to the top film [7]. Illustrative results shown in Fig. 6d suggest that even a modest improvement in the permeability of the support solid can impact the performance much more than any other parameter change. In fact, early asymmetric desalination membranes possessed this feature, where the top skin layer was the same material as the underlying porous structure that acted as the support. In that case, the relative diffusivity (in Fig. 6d) would be unity. However, as can be seen, even much smaller values of the relative diffusivity produce a discernible effect. A possibly promising avenue to improve upon the overall performance of the composite structure would therefore be to make the support more 'transparent' in terms of its obstructing properties, leaving its function to be purely mechanical, as originally intended. However, to do so in practice may prove to be challenging from a materials and fabrication standpoint, as compatibility between the overlaying film and underlying support and the effect on the interfacial polymerization reaction are not yet clearly understood.

\subsection{Effect of 'voids' in the thin film}

We now turn to consider the structure of the overlaying thin film, the part of the composite responsible for the actual separation (in the case of liquids; for gases, the 'gutter' layer, added so as to reduce the restriction imposed by the support, as well as the support may also affect separation properties - see, for example, [41, 45] ). Recent work has shown, through improved resolution and careful use of SEM and TEM imaging techniques as well as other methods, that the structure of aromatic polyamide thin films is not homogeneous and contains distinctive voids [48 52] (see Fig. 7a,b). Furthermore it was shown experimentally that these voids are, at least in part, connected directly to the permeate space. This was shown indirectly, by illustrating the presence of holes in the back surface of the polyamide layer [2, 50, 53], where these holes presumably connect 
closed-off voids with the permeate space. This was further corroborated experimentally by back-filtration of nanoparticles, introduced through the support side of a composite membrane [54] (see Fig. 76). These were then imaged using TEM, and found deposited within the inner surface of the voids, illustrating their connectivity with the permeate space as well as their separation from the feed space.

This new insight on the structure and resulting transport pathways has yet to be comprehensively studied theoretically. Recently, this has been studied by Wong et al. [55] and Lin et al. [56], where the diffusion through a composite membrane was considered, including the presence of voids, modelled as inclusions with a large diffusivity compared with that of the surrounding material. The effect of the size and overall fraction of voids in the film was considered, with particular attention paid to the roughness, possibly resulting from the presence of these voids. Lin et al. [56] concluded that the main contribution to the overall transport in the thin film is dominated by the diffusivity, rather than the void fraction or partition coefficient. However, this calculation did not consider the possible direct connectivity of voids with the permeate space, nor the relative position of the voids with respect to the support pores. In such cases, Wong et al. [55] showed that the relative location of the voids within the film may have a large impact on the permeability, particularly when the voids are well-connected with the permeate space. However, only a minor improvement is possible if the voids are not in direct communication with the underlying support pores; in the presence of a 'base film' covering the support pores, the overlaying voids are of little impact [55, 56]. In contrast, when there is no such base film and the voids are well-connected to the permeate space, the impact of the voids becomes pronounced and a clear theoretical correlation appears between the observable roughness of the film, and the permeance of the composite (see Fig. $7 \mathrm{~d}$ ). This correlation has been a long-debated feature in the experimental literature, which has often shown inconsistent trends of roughness-permeability. Under the conditions described above, the increased permeance may be readily attributed to the larger surface area of the undulating film compared with a flat one. When morphology is well-controlled and the film is thin enough to be considered a continuous phase, there is clear experimental evidence that increasing the surface area by 'crumpling' is a good strategy for increasing permeance [57, 58]. Further refinement of the experimental characterization of these morphological features, accompanied by modelling of the impact on transport, are likely to prove important steps 
(a)

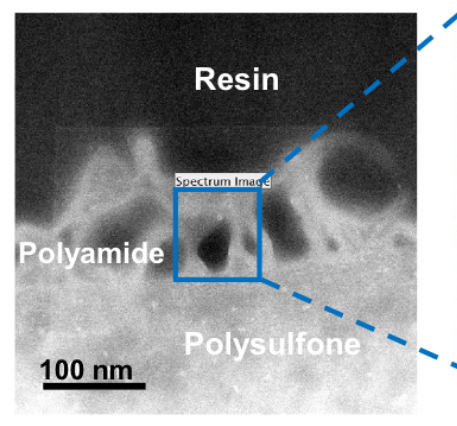

(c)

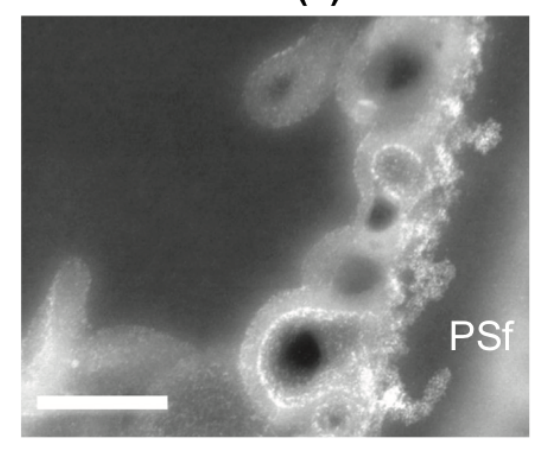

(b)

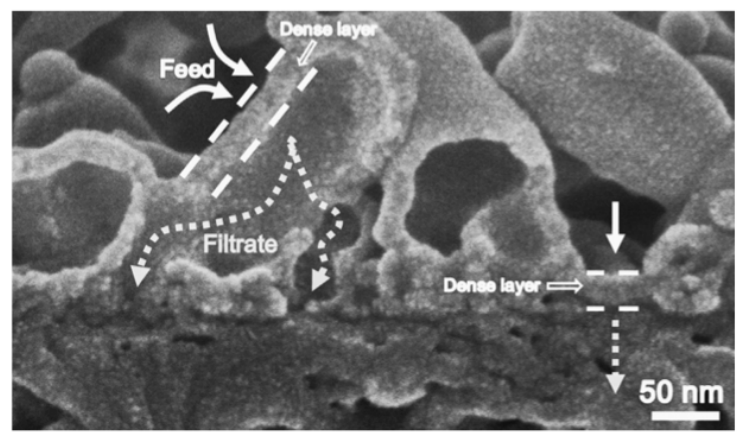

(d)

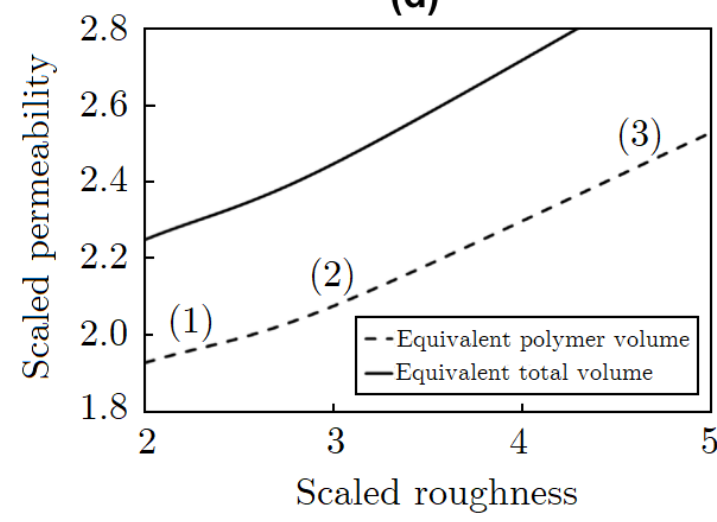

(1)

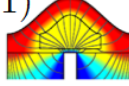

(2)

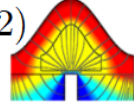

(3)

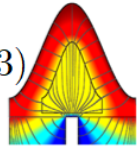

Figure 7: Effect of 'voids' in the thin film on transport. (a) Elemental TEM section of an isolated polyamide film, showing nitrogen-containing red regions identified as polyamide enclosing dark 'void' regions. Taken from [48]. (b) Cross-sectional SEM image of a polyamide film, illustrating potential pathways from the feed side and into voids connecting to the support, permeate side. Taken from [50]. (c) Back-filtration experiment with stained nanoparticles, shown lodged within 'voids', on the supportside of the polyamide film (the direction from which they came with the filtered feed). Taken from [54]. (d) Model calculations of the increased trend in relative permeance with the film roughness, when voids (correlated with the roughness) are contained in the film and are well connected with the permeate space. numbered points refer to the model structure on the right, where the concentration field and diffusion pathlines are also shown. Taken from [55].

in advancing better design.

\subsection{A note on selectivity and rejection in composite membranes}

An important thing to remember is that, to good approximation, all the morphological features considered in the previous section equally affect all diffusing species, and so do not change the intrinsic selectivity of the membrane. This is expected to hold true provided the assumption of decoupled transport is valid. However, some impact on the observed rejection can theoretically occur and is worth a brief mention. In the case of 
a solvent/solute separation, where the solvent transport is dominated by an externally controlled applied pressure while the solute transport is driven primarily by the concentration difference, it can be shown that the solute rejection, a common measure of process selectivity, may be modified by the membrane morphology. This is a consequence of the ability to fix the solvent flux (albeit at a possible energetic penalty if a larger pressure is required) while modifying the solute flux [7]. Analytically, it has been shown asymptotically that the rejection $\left(R_{s}\right)$ depends on support properties (pore radius and porosity) according to the relation 44

$$
R_{s} \sim \frac{1}{1+\phi / \tilde{r}}
$$

showing that increasing the pore size, for a given porosity, will increase the rejection. The implication is that one may potentially tune the rejection of a membrane through manipulation of support morphology, offering an extra degree of freedom, beyond the structure and chemistry of the top layer.

\section{Conclusions}

In this discussion paper we have set out to outline the forefronts in membrane structureperformance modelling, considering two broad classifications of membrane: porous and composite. In each case we quantified the membrane performance through the permeance and, where appropriate, selectivity. We first examined porous membranes, establishing conditions for which the surface porosity impacts the overall permeance of the membrane. The impact on performance of the surface layer as the pore size and pore spatial distribution are varied, was quantified. Periodic, isoporous configurations were found to have the largest permeance (with the particular periodic structure, e.g., square or hexagonal, playing only a small role on the performance). Further, two types of depth structures were considered - granular and cellular - showing how granular layers provide an improved permeance. While this must be considered alongside the corresponding structural integrity, it nevertheless uncovers a potentially promising route to designing improved membranes. Finally, we considered membrane selectivity and showed how this is affected by surface porosity and its distribution; moreover, it was demonstrated that, by considering capture within the membrane, the selectivity can be tuned, particularly when one can design membranes with a graded porosity with depth. 
In a similar spirit, we then turned our attention to composite membranes and examined the effect of the pore size and spatial distribution of the support on the overall composite permeance. Here we showed how the support pore size and porosity impact the permeance with the maximum permeance achieved with small pores and large porosity. Although the spatial distribution of pores had some impact, this was not dramatic (generally, at most a 20\% difference). This suggests that, while an increased porosity of the support is desirable to improve permeance, there is no significant advantage to switching to a periodic, isoporous supports. Another strategy for decreasing the impact of the support is by fabricating this from a material that permits some diffusion of the permeating species through the solid phase. Pores or voids within the top layer of the composite can dramatically impact the permeance, assuming that they are connected with the permeate space; if correlated with increased surface roughness they contribute to a higher permeance through increased surface area.

An overarching theme of this paper was model simplicity. While this strategy offers a great deal of value in understanding seemingly complex processes, more elaborate modelling can provide a much more detailed picture. Ideally, the simple models that have been laid out here serve as a route to sweeping the parameter space to determine the areas in which effort should be focused. From here, more detailed modelling techniques can provide a way of fine-tuning the results, alongside actual experimentation. One notable avenue in need of further study is the development of a more quantitative method for calculating the permeance of a porous membrane where a fraction of the membrane leads to dead ends. While such membranes are prevalent, strategies that determine from a surface of pores how many are actually active are not well established. A second area of study concerns the hindered transport of particles through a membrane. Here, the retardation rather than trapping of particles may be used as a separation tool. Incorporating the notion of porosity-graded filters introduced in this perspective paper could lead to interesting new avenues for study in this area.

We hope that this discussion paper has laid the groundwork for future studies in which mathematical modelling efforts and experimental techniques are unified to drive the forefronts in membrane separation. 


\section{Nomenclature}

$\tilde{\boldsymbol{v}} \quad$ Dimensionless fluid velocity flow field

$\boldsymbol{v} \quad$ Fluid velocity flow field, $\mathrm{m} / \mathrm{s}$

$\tilde{A}_{m} \quad$ Dimensionless membrane area, scaled as $A_{m}=A_{m} L^{* 2}$

$\tilde{h}_{\text {bottom }}$ Dimensionless thickness of the membrane bottom layer scaled with $L^{*}$

$\tilde{h}_{\text {top }} \quad$ Dimensionless thickness of the membrane top layer scaled with $L^{*}$

$\tilde{k} \quad$ Dimensionless membrane hydraulic permeability, scaled as $k=\tilde{k} L^{*} / m u$

$\tilde{P} \quad$ Dimensionless hydrodynamic pressure, scaled as $P=\tilde{P} \mu v_{0} / L^{*}$

$\tilde{Q} \quad$ Dimensionless volumetric flow rate, scaled as $Q=\tilde{Q} v_{0} L^{* 2}$

$\tilde{r}_{\text {bottom }}$ Dimensionless radius of the membrane pore in the bottom layer scaled with $L^{*}$

$\tilde{r}_{\text {top,avg }}$ Dimensionless average pore radius in the membrane top layer

$\tilde{r}_{t o p} \quad$ Dimensionless radius of the membrane pore in the top layer scaled with $L^{*}$

$A_{m} \quad$ Membrane area, $m^{2}$

$C_{f, s}$ Concentration of the species in the film (subscript $f$ ) and in the support (subscript $\mathrm{s}), \mathrm{kg} / \mathrm{m}^{3}$

$D_{f, s}$ Diffusivity of the species in the film (subscript $f$ ) and in the support (subscript s), $m^{2} / s$

$H \quad$ Dimensionless thickness of the film, scaled as $H=h / r$

$h \quad$ Thickness of the membrane, $m$

$h_{\text {bottom }}$ Thickness of the membrane bottom layer, $m$

$h_{\text {top }} \quad$ Thickness of the membrane top layer, $m$

$k \quad$ Membrane hydraulic permeability, $m /$ Pa.s 
$k_{r} \quad$ Relative permeance as defined in Eq. 10

$L^{*} \quad$ Typical length scale as the distance between the pores, $m$

$L_{p} \quad$ Same as k; Membrane hydraulic permeability, $m / P a . s$

$P \quad$ Hydrodynamic pressure, $P a$

$Q \quad$ Volumetric flow rate, $\mathrm{m}^{3} / \mathrm{s}$

$r \quad$ Radius of the membrane pore, $m$

$r_{p} \quad$ Radius of the particle rejected, $m$

$R_{s} \quad$ Membrane solute rejection

$r_{\text {bottom }}$ Radius of the membrane pore in the bottom layer, $m$

$r_{\text {top,avg }}$ Average pore radius in the membrane top layer, $m$

$r_{\text {top }} \quad$ Radius of the membrane pore in the top layer, $m$

$S \quad$ Sieving coefficient, as defined by Eq. (5)

$v_{0} \quad$ Typical velocity scale, $\mathrm{m} / \mathrm{s}$

\section{Greek symbols}

$\Delta P \quad$ Transmembrane pressure drop across the membrane, $P a$

$\lambda \quad$ Size ratio of the particle being rejected $\left(r_{p}\right)$ and the membrane pore radius $(r)$

$\mu \quad$ Viscosity of the solution filtered, Pa.s

$\phi_{\text {bottom }}$ Porosity of the bottom layer

$\phi_{\text {top }} \quad$ Porosity of the top layer

$\sigma \quad$ Standard deviation in the membrane pore size distribution

$\xi \quad$ constant dependent on the geometrical layout of the pores 


\section{References}

[1] A. K. Ghosh, E. M. V. Hoek, Impacts of support membrane structure and chemistry on polyamide-polysulfone interfacial composite membranes, J. Membr. Sci. 336 (1-2) (2009) 140-148. doi:10.1016/j.memsci.2009.03.024.

[2] F. A. Pacheco, I. Pinnau, M. Reinhard, J. O. Leckie, Characterization of isolated polyamide thin films of RO and NF membranes using novel TEM techniques, Journal of Membrane Science 358 (1-2) (2010) 51-59. doi:10.1016/j.memsci.2010.04.032.

[3] A. Tiraferri, N. Y. Yip, W. A. Phillip, J. D. Schiffman, M. Elimelech, Relating performance of thin-film composite forward osmosis membranes to support layer formation and structure, J. Membr. Sci. 367 (1-2) (2011) 340-352. doi:10.1016/j . memsci.2010.11.014.

[4] J. Hahn, J. I. Clodt, V. Filiz, V. Abetz, Protein separation performance of selfassembled block copolymer membranes, RSC Adv. 4 (2014) 10252. doi:10.1039/ c3ra47306f.

[5] M. Mulder, Basic Principles of Membrane Technology, Kluwer Academic Publishers, Dordrecht, Boston, London, 1992.

[6] H. Strathmann, Introduction to Membrane Science and Technology, Wiley, 2011.

[7] G. Z. Ramon, M. C. Wong, E. M. Hoek, Transport through composite membrane, part 1: Is there an optimal support membrane?, J. Membr. Sci. 415-416 (2012) 298-305.

[8] J. Wijmans, P. Hao, Influence of the porous support on diffusion in composite membranes, J. Membr. Sci. 494 (2015) 78-85. doi:10.1016/j.memsci.2015.07.047.

[9] L. Zhu, W. Jia, M. Kattula, K. Ponnuru, E. P. Furlani, H. Lin, Effect of porous supports on the permeance of thin film composite membranes: Part I. Track-etched polycarbonate supports, J. Membr. Sci. (2015) 1-12.

[10] Z. Jiang, S. Karan, A. G. Livingston, Water Transport through Ultrathin Polyamide Nanofilms Used for Reverse Osmosis, Adv. Mater. 30 (15) (2018) 1-7. doi:10.1002/ adma.201705973. 
[11] V. Abetz, Isoporous block copolymer membranes., Macromol. Rapid Comm. 36 (1) (2015) 10-22. doi:10.1002/marc.201400556.

[12] Y. Zhang, J. L. Sargent, B. W. Boudouris, W. A. Phillip, Nanoporous membranes generated from self-assembled block polymer precursors: Quo Vadis?, J. Appl. Polym. Sci. 132 (21). doi:10.1002/app.41683.

[13] H. D. Tong, H. V. Jansen, V. J. Gadgil, C. G. Bostan, E. Berenschot, C. J. Van Rijn, M. Elwenspoek, Silicon Nitride Nanosieve Membrane, Nano Lett. 4 (2) (2004) 283-287. doi:10.1021/n10350175.

[14] C. C. Striemer, T. R. Gaborski, J. L. McGrath, P. M. Fauchet, Charge- and size-based separation of macromolecules using ultrathin silicon membranes, Nature 445 (7129) (2007) 749-753. doi:10.1038/nature05532.

[15] B. J. Hinds, N. Chopra, T. Rantell, R. Andrews, V. Gavalas, L. G. Bachas, Aligned multiwalled carbon nanotube membranes., Science (New York, N.Y.) 303 (5654) (2004) 62-65. doi:10.1126/science.1092048.

[16] H. Li, Z. Song, X. Zhang, Y. Huang, S. Li, Y. Mao, H. J. Ploehn, Y. Bao, M. Yu, Ultrathin, molecular-sieving graphene oxide membranes for selective hydrogen separation, Science 342 (6154) (2013) 95-98. arXiv:1005.0853, doi:10.1126/science. 1236686 .

[17] C. Tang, Y. Zhao, R. Wang, C. Hélix-Nielsen, A. Fane, Desalination by biomimetic aquaporin membranes: Review of status and prospects, Desalination 308 (2013) 34-40. doi:10.1016/j.desal.2012.07.007.

[18] J. Lee, J. H. Jang, H.-R. Chae, S. H. Lee, C.-H. Lee, P.-K. Park, Y.-J. Won, I.C. Kim, A facile route to enhance the water flux of a thin-film composite reverse osmosis membrane: incorporating thickness-controlled graphene oxide into a highly porous support layer, J. Mater. Chem. A 3 (44) (2015) 22053-22060. doi:10.1039/ C5TA04042F.

[19] M. E. Suk, N. R. Aluru, Modeling Water Flow Through Carbon Nanotube Membranes with Entrance/Exit Effects, Nanosc. Microsc. Therm. Eng. 21 (4) (2017) 247-262. doi:10.1080/15567265.2017.1355949. 
[20] M. Shi, G. Printsypar, O. Iliev, V. M. Calo, G. L. Amy, S. P. Nunes, Water flow prediction for membranes using 3D simulations with detailed morphology, J. Membr. Sci. 487 (2015) 19-31. doi:10.1016/j.memsci.2015.03.036.

[21] H. L. Weissberg, End correction for slow viscous flow through long tubes, Phys. Fluids 5 (9) (1962) 1033-1036. arXiv:arXiv:1011.1669v3, doi:10.1063/1.1724469.

[22] R. A. Sampson, On Stokes' current function, Phil. Trans. Royal Soc. London. A 182 (1891) 449-518.

[23] L. G. Leal, Advanced transport phenomena: fluid mechanics and convective transport processes, Cambridge University Press, 2007.

[24] P. Aimar, M. Meireles, V. Sanchez, A contribution to the translation of retention curves into pore size distributions for sieving membranes, J. Membr. Sci. 54 (3) (1990) 321-338. doi:10.1016/S0376-7388(00)80618-3.

[25] A. Mehta, A. L. Zydney, Permeability and selectivity analysis for ultrafiltration membranes, J. Membr. Sci. 249 (1-2) (2005) 245-249. doi:10.1016/j.memsci. 2004.09.040.

[26] D. M. Kanani, W. H. Fissell, S. Roy, A. Dubnisheva, A. Fleischman, A. L. Zydney, Permeability-selectivity analysis for ultrafiltration: Effect of pore geometry, J. Membr. Sci. 349 (1-2) (2010) 405-410. doi:10.1016/j.memsci.2009.12.003.

[27] K. H. Jensen, A. X. C. N. Valente, H. A. Stone, Flow rate through microfilters: Influence of the pore size distribution, hydrodynamic interactions, wall slip, and inertia, Phys. Fluid 26 (5) (2014) 052004. doi:10.1063/1.4876937.

[28] A. L. Zydney, P. Aimar, M. Meireles, J. M. Pimbley, G. Belfort, Use of the log-normal probability density function to analyze membrane pore size distributions: functional forms and discrepancies, J. Membr. Sci.doi:10.1016/0376-7388(94)80090-1.

[29] T. H. Young, L. P. Cheng, D. J. Lin, L. Fane, W. Y. Chuang, Mechanisms of PVDF membrane formation by immersion-precipitation in soft (1-octanol) and harsh (water) nonsolvents, Polymer 40 (19) (1999) 5315-5323. 
[30] V. Abetz, Isoporous block copolymer membranes., Macromolecular rapid communications 36 (1) (2015) 10-22. doi:10.1002/marc.201400556.

[31] C. C. Ho, A. L. Zydney, Effect of membrane morphology on the initial rate of protein fouling during microfiltration, J. Membr. Sci. 155 (2) (1999) 261-275. doi: 10.1016/S0376-7388(98)00324-X

[32] W. M. Deen, Hindered Transport of Large Molecules in Liquid-Filled Pores, AIChE J. 33 (9) (1987) 1409-1425. doi:10.1002/aic.690330902.

[33] P. Dechadilok, W. M. Deen, Hindrance factors for diffusion and convection in pores, Ind. Eng. Chem. Res. 45 (21) (2006) 6953-6959. doi:10.1021/ie051387n.

[34] J. D. Ferry, Statistic evaluation of sieve constants in ultrafiltration, J. Gen. Physiol. 20 (1936) 95-104. doi:10.1085/jgp.20.1.95.

[35] M. P. Dalwadi, I. M. Griffiths, M. Bruna, Understanding how porosity gradients can make a better filter using homogenization theory, Proc. Royal Soc. London A: Math. Phys. Eng. Sci. 471 (2182). doi:10.1098/rspa.2015.0464.

[36] H. K. Lonsdale, Transport properties of cellulose acetate osmotic membranes., U. Merten and R. L. Riley. J. Appl. Polymer Sci. 9 (4) (1965) 1341-1362.

[37] J. G. Wijmans, R. W. Baker, The solution-diffusion model: a review, J. Membr. Sci. 107 (1-2) (1995) 1-21. doi:10.1016/0376-7388(95)00102-I.

[38] H. K. Lonsdale, R. L. Riley, C. R. Lyons, D. P. Carosella, Transport in Composite Reverse Osmosis Membranes, Springer US, Boston, MA, 1971, pp. 101-122. doi: 10.1007/978-1-4684-1911-5_6.

[39] A. M. J. Davis, C. R. Ethier, Transport through materials bounded by porous surfaces, Chem. Eng. Sci. 48 (9) (1993) 1655-1663.

[40] J. L. Lopez, S. L. Matson, J. Marchese, J. A. Quinn, Diffusion through composite membranes: A two-dimensional analysis, J. Membr. Sci. 27 (3) (1986) 301-325.

[41] U. Beuscher, C. H. Gooding, The influence of the porous support layer of composite membranes on the separation of binary gas mixtures, J. Membr. Sci. 152 (1) (1999) 99-116. 
[42] G. Z. Ramon, E. M. V. Hoek, Transport through composite membranes, part 2 : Impacts of roughness on permeability and fouling, J. Membr. Sci. 425-426 (2013) $141-148$.

[43] M. F. Jimenez-Solomon, P. Gorgojo, M. Munoz-Ibanez, A. G. Livingston, Beneath the surface: Influence of supports on thin film composite membranes by interfacial polymerization for organic solvent nanofiltration, J. Membr. Sci. 448 (2013) 102-113. doi:10.1016/j.memsci.2013.06.030.

[44] M. Bruna, S. J. Chapman, G. Z. Ramon, The effective flux through a thin-film composite membrane, EPL (Europhys. Lett.) 110 (4) (2015) 40005. doi:10.1209/ 0295-5075/110/40005.

[45] M. Kattula, K. Ponnuru, L. Zhu, W. Jia, H. Lin, E. P. Furlani, Designing ultrathin film composite membranes: the impact of a gutter layer., Scientific Rep. 5 (2015) 15016. doi:10.1038/srep15016.

[46] S. Manickam, G. Z. Ramon, J. McCutcheon, Modeling the effect of film-pore coupled transport on composite forward osmosis membrane performance, J. Membr. Sci. 523. doi:10.1016/j.memsci.2016.09.043.

[47] A. Ghadimi, S. Norouzbahari, H. Lin, H. Rabiee, B. Sadatnia, Geometric restriction of microporous supports on gas permeance efficiency of thin film composite membranes, J. Membr. Sci. 563 (2018) 643-654. doi:10.1016/j.memsci.2018.06.025.

[48] L. Lin, R. Lopez, G. Z. Ramon, O. Coronell, Investigating the void structure of the polyamide active layers of thin-film composite membranes, J. Membr. Sci. 497 (2016) 365-376. doi:10.1016/j.memsci.2015.09.020.

[49] F. Pacheco, R. Sougrat, M. Reinhard, J. O. Leckie, I. Pinnau, 3D visualization of the internal nanostructure of polyamide thin films in RO membranes, J. Membr. Sci. 501 (2015) 33-44. doi:10.1016/j.memsci.2015.10.061.

[50] H. Yan, X. Miao, J. Xu, G. Pan, Y. Zhang, Y. Shi, M. Guo, Y. Liu, The porous structure of the fully-aromatic polyamide film in reverse osmosis membranes, J. Membr. Sci. 475 (2015) 504-510. doi:10.1016/j.memsci.2014.10.052. 
[51] M. M. Kłosowski, C. M. McGilvery, Y. Li, P. Abellan, Q. Ramasse, J. T. Cabral, A. G. Livingston, A. E. Porter, Micro-to nano-scale characterisation of polyamide structures of the SW30HR RO membrane using advanced electron microscopy and stain tracers, J. Membr. Sci. 520 (2016) 465-476.

[52] X. Lu, S. Nejati, Y. Choo, C. O. Osuji, J. Ma, M. Elimelech, Elements Provide a Clue: Nanoscale Characterization of Thin-Film Composite Polyamide Membranes, ACS Appl. Mater. Interfaces 7 (31) (2015) 16917-16922. doi:10.1021/acsami.5b05478.

[53] J. Xu, H. Yan, Y. Zhang, G. Pan, Y. Liu, The morphology of fully-aromatic polyamide separation layer and its relationship with separation performance of TFC membranes, J. Membr. Sci. 541 (2017) 174-188. doi:10.1016/j.memsci.2017.06. 057.

[54] Y. Li, M. M. Kłosowski, C. M. McGilvery, A. E. Porter, A. G. Livingston, J. T. Cabral, Probing flow activity in polyamide layer of reverse osmosis membrane with nanoparticle tracers, J. Membr. Sci. 534 (April) (2017) 9-17. doi:10.1016/j. memsci.2017.04.005.

[55] M. C. Wong, L. Lin, O. Coronell, E. M. Hoek, G. Z. Ramon, Impact of liquid-filled voids within the active layer on transport through thin-film composite membranes, J. Membr. Sci. 500 (2016) 124-135. doi:10.1016/j.memsci.2015.11.033.

[56] L. Lin, T. M. Weigand, M. W. Farthing, P. Jutaporn, C. T. Miller, O. Coronell, Relative importance of geometrical and intrinsic water transport properties of active layers in the water permeability of polyamide thin-film composite membranes, J. Membr. Sci. 564 (2018) 935-944.

[57] S. Karan, Z. Jiang, A. G. Livingston, Sub10 nm polyamide nanofilms with ultrafast solvent transport for molecular separation, Science 348 (6241) (2015) 1347-1351. doi:10.1126/science.aaa5058.

[58] Z. Tan, S. Chen, X. Peng, L. Zhang, C. Gao, Polyamide membranes with nanoscale Turing structures for water purification, Science 360 (6388) (2018) 518-521. doi: 10.1126/science. aar6308. 